\title{
REVIEW ARTICLE \\ Systematic review of risk prediction scores for surgical site infection or periprosthetic joint infection following joint arthroplasty
}

\author{
S. K. KUNUTSOR*, M. R. Whitehouse, A. W. BLOM and A. D. BESWICK \\ Musculoskeletal Research Unit, School of Clinical Sciences, University of Bristol, Learning \& Research Building \\ (Level 1), Southmead Hospital, Southmead Road, Bristol, BS10 5NB, UK
}

Received 30 November 2016; Final revision 2 February 2017; Accepted 5 February 2017; first published online 7 March 2017

\section{SUMMARY}

Accurate identification of individuals at high risk of surgical site infections (SSIs) or periprosthetic joint infections (PJIs) influences clinical decisions and development of preventive strategies. We aimed to determine progress in the development and validation of risk prediction models for SSI or PJI using a systematic review. We searched for studies that have developed or validated a risk prediction tool for SSI or PJI following joint replacement in MEDLINE, EMBASE, Web of Science and Cochrane databases; trial registers and reference lists of studies up to September 2016. Nine studies describing 16 risk scores for SSI or PJI were identified. The number of component variables in a risk score ranged from 4 to 45 . The $\mathrm{C}$-index ranged from 0.56 to $0 \cdot 74$, with only three risk scores reporting a discriminative ability of $>0 \cdot 70$. Five risk scores were validated internally. The National Healthcare Safety Network SSIs risk models for hip and knee arthroplasties (HPRO and KPRO) were the only scores to be externally validated. Except for HPRO which shows some promise for use in a clinical setting (based on predictive performance and external validation), none of the identified risk scores can be considered ready for use. Further research is urgently warranted within the field.

Key words: Bone infections, epidemiology, risk assessment.

\section{INTRODUCTION}

Surgical site infections (SSIs) which can be classified as superficial wound infections, deep wound infections, or periprosthetic joint infections (PJIs) [1], are uncommon but serious complications of total joint replacements [2, 3]. PJIs can result in severe pain, functional deficits and even death [4-6]; and their management is a huge financial burden to health care systems [7, 8]. With increasing life expectancy and a growing indication for primary joint replacements

\footnotetext{
* Author for correspondence: S. K. Kunutsor, Musculoskeletal Research Unit, School of Clinical Sciences, University of Bristol, Learning \& Research Building (Level 1), Southmead Hospital, Southmead Road, Bristol, BS10 5NB, UK.

(Email: setor.kunutsor@bristol.ac.uk)
}

[9], there will be a proportionate rise in the number of patients who will be affected by PJIs. An approach to tackle the increasing incidence of PJIs is to identify those people at high risk and offer appropriate interventions. Early and accurate identification of individuals at high risk of PJI influences clinical decisions and development of targeted preventive strategies, and helps to optimise resources required for detection of PJI. Several factors such as characteristics of the patient, surgical procedure and postoperative care, have been found to influence the risk of developing PJI $[10,11]$, however their potential utility for PJI risk assessment remains uncertain.

A risk score or prognostic model is a statistical equation that predicts an individual's disease risk based on a combination of the values of multiple 
predictors or risk factors [12]. Risk prediction scores are ideally developed using data from long-term follow-up of large population-based cohorts of individuals without a history of the event of interest (SSI or PJI in this case) at baseline. The dataset is used to identify important predictors and the model equation is developed [13]. Using the derivation sample, the score's apparent performance is evaluated in a process known as internal evaluation. The next stage is external validation, which examines the generalisability of the model using new data. Risk prediction scores first emerged in the area of cardiovascular disease (CVD) prevention and have been widely used globally in clinical and public health practice. Well known amongst them is the Framingham CVD risk score [14] (a risk score which assesses an individual's risk of a cardiovascular event within 10 years), which is a commonly used algorithm in clinical practice and accepted tool in preventive medicine.

Prevention of SSIs or PJI is a high policy priority and there has been an increasing interest in the development of risk prediction tools for SSI or PJI over the last decade. However, unlike the substantial progress made in CVD prevention using risk scores, the amount of progress made in the area of SSIs or PJIs is uncertain. There is therefore a need for objective data on the development of risk scores (including their component variables), their discriminative abilities, whether they have been externally validated, and whether their clinical effectiveness have been assessed in well-designed randomised controlled trials (RCTs). In this context, using systematic review methodology, we aimed to: (i) identify and summarise studies reporting the development of risk prediction scores for SSI or PJI; (ii) assess clinical variables selected for model inclusion and the predictive performance of these models; (iii) assess if identified models have been externally validated and their performances compared; (iv) assess if the impact or clinical effectiveness of these risk scores have been evaluated in appropriate RCTs and (v) finally to identify gaps in the existing evidence and whether further research is needed in the field.

\section{METHODS}

This review was conducted using a predefined protocol, which has been registered in the PROSPERO prospective register of systematic reviews (CRD42016042158), and in line with PRISMA guidelines [15] (Supplementary Material 1). We searched MEDLINE, EMBASE,
Web of Science and the Cochrane Library electronic databases up to 30 September 2016. The publicly available trial registers ClinicalTrials.gov, UKCRN (UK Clinical Research Network) Study Portfolio Database, and the WHO International Clinical Trials Registry Platform were also searched. The search strategy combined free and $\mathrm{MeSH}$ search terms and combination of key words relating to risk prediction (e.g., 'predict', 'risk score', 'sensitivity'), SSI or PJI (e.g., 'periprosthetic joint infection', 'deep infection', 'surgical site infection'), and joint replacement (e.g., 'hip replacement', 'knee replacement', 'hip arthroplasty', 'knee arthroplasty'). No restrictions were placed on publication dates and only articles published in English were considered. Reference lists of retrieved articles and relevant review articles identified on the topic were manually scanned for all relevant additional studies. Detailed description of all Materials and Methods, as well as the Literature Search Strategy are available in Supplementary Materials 2 and 3.

\section{RESULTS}

\section{Study identification and selection}

Figure 1 shows the flow of studies through the review. Our literature search strategy identified 1802 potentially relevant articles. After the initial screening of titles and abstracts, 15 articles remained for further evaluation. Following detailed evaluation which included full-text reviews, six articles were excluded because (i) they were studies of diagnostic scores $(n=2)$ and (ii) they were studies of risk scores for outcomes such as readmission, infection eradication and treatment outcome of PJI $(n=4)$. The remaining nine articles met the inclusion criteria and were included in the review [16-24].

\section{Study characteristics and quality assessment}

Table 1 summarises characteristics of the studies in the sample. Studies were published between 2006 and 2016, with all but one appearing in 2011-2016. One study was reported as a published conference abstract [16]. Overall, the studies involved 482877 joint replacements, including 6968 SSIs or PJIs. For studies that reported age data, the baseline age of participants ranged from 56 to 81 years. The sample size of cohorts ranged from 217 to 172055 and follow-up for infection outcomes ranged from 30 days to 2 years. For 

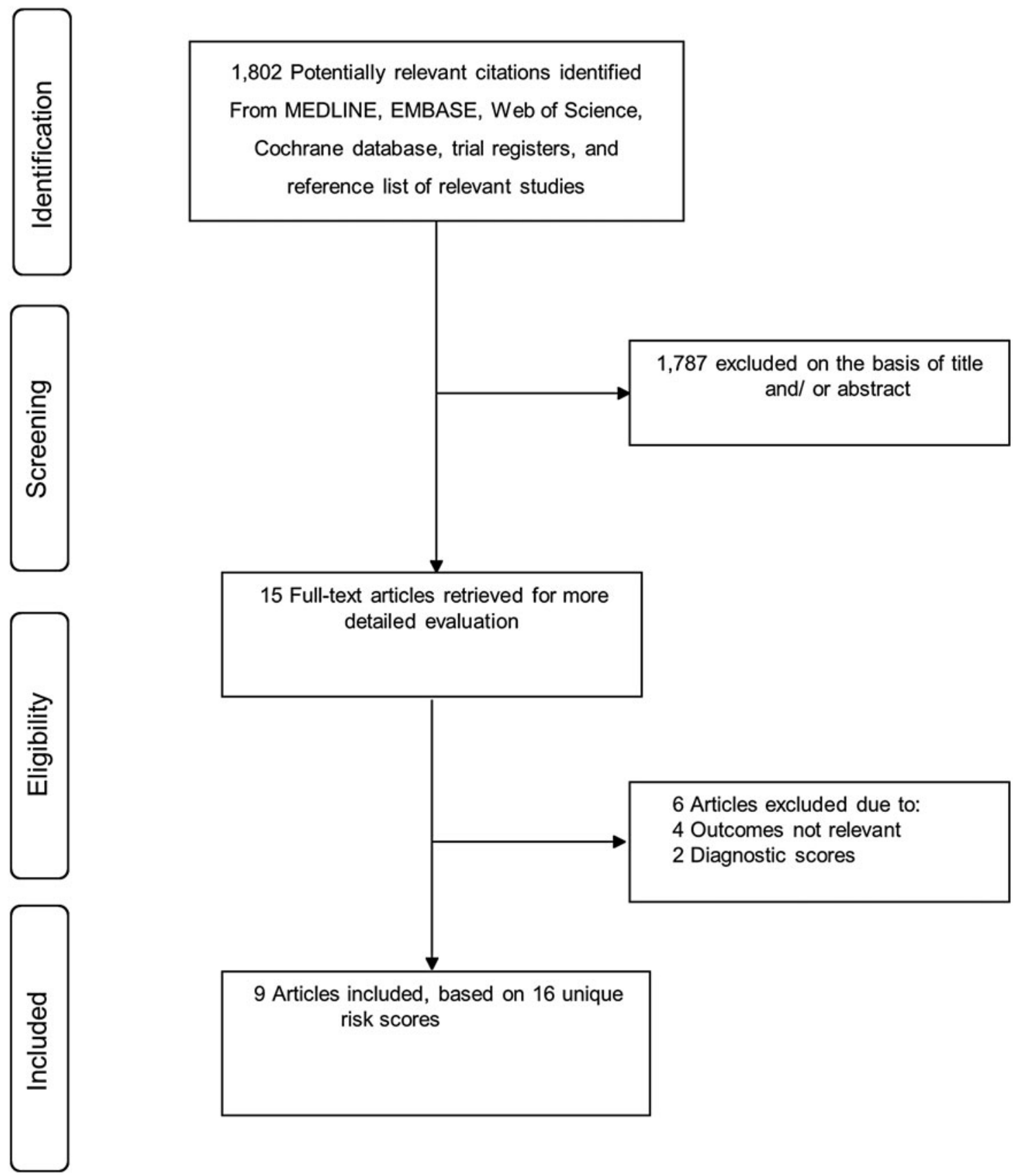

Fig. 1. PRISMA flow diagram.

the assessment of infections, the majority of the studies used Centre for Disease Control or Infectious Diseases Society of America criteria. Studies classified infection outcomes as SSI or PJI specifically. One study employed both SSI and PJI outcomes [21] and another study used PJI recurrence [24]. Quality assessment using PROBAST showed evidence of high overall risk of bias throughout the included studies. Five risk scores had unclear concern for overall applicability and only two scores were deemed to be usable in the targeted individuals and context (the National Healthcare Safety Network (NHSN) SSIs risk models for hip and knee arthroplasties (HPRO and KPRO)) [18] (Supplementary Material 4).

\section{Model description and development}

Table 2 provides details of risk scores included in eligible studies: their component predictors, statistical properties, measures of discrimination and/or calibration, and 
Table 1. Summary characteristics of studies included in the review

\begin{tabular}{|c|c|c|c|c|c|c|c|c|c|c|c|}
\hline $\begin{array}{l}\text { Lead author, } \\
\text { publication } \\
\text { date }\end{array}$ & Location & $\begin{array}{l}\text { Baseline } \\
\text { year }\end{array}$ & $\begin{array}{l}\text { Study } \\
\text { design }\end{array}$ & $\begin{array}{l}\text { Population/sampling } \\
\text { frame }\end{array}$ & $\begin{array}{l}\text { Mean/median } \\
\text { age }\end{array}$ & Name of risk tool & $\begin{array}{l}\text { Specific outcome } \\
\text { reported }\end{array}$ & Sample size & $\begin{array}{l}\text { Number of } \\
\text { events }\end{array}$ & $\begin{array}{l}\text { Duration of } \\
\text { follow-up }\end{array}$ & $\begin{array}{l}\text { Ascertainment of } \\
\text { outcome (s) }\end{array}$ \\
\hline $\begin{array}{l}\text { Geubbels } \\
\text { et al. [17] }\end{array}$ & $\begin{array}{l}\text { The } \\
\text { Netherlands }\end{array}$ & 1996-2000 & $\begin{array}{l}\text { Prospective } \\
\text { cohort }\end{array}$ & $\begin{array}{l}\text { Patients who underwent } \\
\text { primary THA in } 62 \\
\text { acute care hospitals. }\end{array}$ & NR & $\begin{array}{l}\text { THA-specific risk } \\
\text { model for SSI }\end{array}$ & SSIs & 13770 & NR & NR & CDC definition \\
\hline $\begin{array}{l}\text { Mu et al. } \\
{[18]}\end{array}$ & USA & 2006-2008 & $\begin{array}{l}\text { Retrospective } \\
\text { cohort }\end{array}$ & $\begin{array}{l}\text { NHSN data } \\
\text { Total primary, partial } \\
\text { primary, partial } \\
\text { revision, total revision } \\
\text { arthroplasty }\end{array}$ & NR & HPRO & $\begin{array}{l}\text { SSI (superficial } \\
\text { incisional, } \\
\text { deep incisional, } \\
\text { and organ/space) } \\
\text { Deep incisional } \\
\text { and organ/space } \\
\text { SSIs }\end{array}$ & 131879 & 1855 & $\begin{array}{l}30 \text { days for } \\
\text { superficial } \\
\text { incisional and } 1 \\
\text { year for deep } \\
\text { incisional and } \\
\text { organ/space } \\
\text { infections }\end{array}$ & CDC definition \\
\hline $\begin{array}{l}\text { Mu et al. } \\
{[18]}\end{array}$ & USA & $2006-2008$ & $\begin{array}{l}\text { Retrospective } \\
\text { cohort }\end{array}$ & $\begin{array}{l}\text { NHSN data } \\
\text { Primary or revision } \\
\text { arthroplasty }\end{array}$ & NR & KPRO & $\begin{array}{l}\text { SSI (superficial } \\
\text { incisional, deep } \\
\text { incisional, and } \\
\text { organ/space) } \\
\text { Deep incisional } \\
\text { and organ/space } \\
\text { SSIs }\end{array}$ & 172055 & 1108 & $\begin{array}{l}30 \text { days for } \\
\text { superficial } \\
\text { incisional and } 1 \\
\text { year for deep } \\
\text { incisional and } \\
\text { organ/space } \\
\text { infections }\end{array}$ & CDC definition \\
\hline $\begin{array}{l}\text { Paxton et al. } \\
{[16]}\end{array}$ & USA & 2001-2009 & $\begin{array}{l}\text { Retrospective } \\
\text { cohort }\end{array}$ & $\begin{array}{l}\text { Kaiser Permanente's } \\
\text { Total Joint } \\
\text { Replacement } \\
\text { Registry. Patients who } \\
\text { underwent total knee } \\
\text { replacement }\end{array}$ & NR & NS & Deep infection & 38094 & 241 & 1 year & CDC definition \\
\hline $\begin{array}{l}\text { Berbari et al. } \\
\text { [19] }\end{array}$ & USA & 2001-2006 & $\begin{array}{l}\text { Prospective } \\
\text { case-control }\end{array}$ & $\begin{array}{l}\text { Patients who underwent } \\
\text { THA or TKA }\end{array}$ & NR & $\begin{array}{l}\text { Baseline Mayo } \\
\text { PJI risk score }\end{array}$ & PJI & 617 & 301 & NR & CDC definition \\
\hline $\begin{array}{l}\text { Berbari et al. } \\
\text { [19] }\end{array}$ & USA & 2001-2006 & $\begin{array}{l}\text { Prospective } \\
\text { case-control }\end{array}$ & $\begin{array}{l}\text { Patients who underwent } \\
\text { THA or TKA }\end{array}$ & NR & $\begin{array}{l}\text { 1-month-postsurgery } \\
\text { Mayo PJI risk score }\end{array}$ & PJI & 574 & 258 & NR & CDC definition \\
\hline $\begin{array}{l}\text { Bozic et al. } \\
{[20]}\end{array}$ & USA & 1998-2009 & $\begin{array}{l}\text { Retrospective } \\
\text { cohort }\end{array}$ & $\begin{array}{l}\text { Medicare patients with } \\
\text { primary THA } \\
\text { (Administrative } \\
\text { claims data) }\end{array}$ & NR & NS & PJI & 53252 & 1102 & 2 years & NR \\
\hline $\begin{array}{l}\text { Lewallen } \\
\text { et al. [21] }\end{array}$ & USA & 2002-2009 & $\begin{array}{l}\text { Retrospective } \\
\text { Cohort }\end{array}$ & $\begin{array}{l}\text { Patients with } \\
\text { procedures performed } \\
\text { at Rochester Mayo } \\
\text { Clinic } \\
\text { Primary or revision } \\
\text { hip replacement }\end{array}$ & $65 \cdot 6$ & HPRO & SSI and PJI & 10869 & $426^{*}$ & 1 year & $\begin{array}{l}\text { Infectious Diseases } \\
\text { Society of America } \\
\text { criteria }\end{array}$ \\
\hline $\begin{array}{l}\text { Lewallen } \\
\text { et al. [21] }\end{array}$ & USA & 2002-2009 & $\begin{array}{l}\text { Retrospective } \\
\text { Cohort }\end{array}$ & $\begin{array}{l}\text { Patients with } \\
\text { procedures performed } \\
\text { at Rochester Mayo } \\
\text { Clinic } \\
\text { Primary or revision } \\
\text { hip replacement }\end{array}$ & $67 \cdot 4$ & KPRO & SSI and PJI & 11072 & $426^{*}$ & 1 year & $\begin{array}{l}\text { Infectious Diseases } \\
\text { Society of America } \\
\text { criteria }\end{array}$ \\
\hline
\end{tabular}


Table 1 (cont.)

\begin{tabular}{|c|c|c|c|c|c|c|c|c|c|c|c|}
\hline $\begin{array}{l}\text { Lead author, } \\
\text { publication } \\
\text { date }\end{array}$ & Location & $\begin{array}{l}\text { Baseline } \\
\text { year }\end{array}$ & $\begin{array}{l}\text { Study } \\
\text { design }\end{array}$ & $\begin{array}{l}\text { Population/sampling } \\
\text { frame }\end{array}$ & $\begin{array}{l}\text { Mean/median } \\
\text { age }\end{array}$ & Name of risk tool & $\begin{array}{l}\text { Specific outcome } \\
\text { reported }\end{array}$ & Sample size & $\begin{array}{l}\text { Number of } \\
\text { events }\end{array}$ & $\begin{array}{l}\text { Duration of } \\
\text { follow-up }\end{array}$ & $\begin{array}{l}\text { Ascertainment of } \\
\text { outcome (s) }\end{array}$ \\
\hline $\begin{array}{l}\text { Inacio } \\
\text { et al. [22] }\end{array}$ & Australia & 2001-2012 & $\begin{array}{l}\text { Retrospective } \\
\text { Cohort }\end{array}$ & $\begin{array}{l}\text { Australian Department } \\
\text { of Veterans' Affairs } \\
\text { database. } \\
\text { Primary unilateral total } \\
\text { hip replacement }\end{array}$ & $80 \cdot 9$ & $\begin{array}{l}\text { RxRisk-V; } \\
\text { Elixhauser; } \\
\text { and Charlson } \\
\text { comorbidities } \\
\text { coding algorithm }\end{array}$ & PJI after THA & 11848 & 364 & 90 days & $\begin{array}{l}\text { ICD-10-AM diagnostic } \\
\text { codes T81·4, T84.5, } \\
\text { T85.79 or } \\
\text { hospitalisations with the } \\
\text { procedure ICD-10-AM } \\
\text { procedure codes } 930301 \\
\text { (lavage of hips); or the } \\
\text { initiation of the } \\
\text { antibiotics gentamicin } \\
\text { (ATC code J01GB03) } \\
\text { or vancomycin (ATC } \\
\text { code J01XA01). }\end{array}$ \\
\hline $\begin{array}{l}\text { Inacio et al. } \\
\text { [22] }\end{array}$ & Australia & 2001-2012 & $\begin{array}{l}\text { Retrospective } \\
\text { Cohort }\end{array}$ & $\begin{array}{l}\text { Australian Department } \\
\text { of Veterans' Affairs } \\
\text { database. } \\
\text { Primary total knee } \\
\text { replacement }\end{array}$ & $79 \cdot 8$ & $\begin{array}{l}\text { RxRisk-V; } \\
\text { Elixhauser; } \\
\text { and Charlson } \\
\text { comorbidities } \\
\text { coding algorithm }\end{array}$ & PJI after TKA & 18972 & 648 & 90 days & $\begin{array}{l}\text { ICD-10-AM diagnostic } \\
\text { codes T81·4, T84.5, } \\
\text { T85.79 or } \\
\text { hospitalisations with the } \\
\text { procedure ICD-10-AM } \\
\text { procedure codes } \\
4950030 \text { (lavage of } \\
\text { knees); or the initiation } \\
\text { of the antibiotics } \\
\text { gentamicin (ATC code } \\
\text { J01GB03) or } \\
\text { vancomycin (ATC code } \\
\text { J01XA01). }\end{array}$ \\
\hline $\begin{array}{l}\text { Maradit } \\
\text { Kremers } \\
\text { et al. [23] }\end{array}$ & USA & 2002-2009 & $\begin{array}{l}\text { Retrospective } \\
\text { cohort }\end{array}$ & $\begin{array}{l}\text { Patients who underwent } \\
\text { primary or revision } \\
\text { THA at Rochester } \\
\text { Mayo Clinic }\end{array}$ & $64 \cdot 6$ & $\begin{array}{c}\text { Claims-based risk } \\
\text { model for THA }\end{array}$ & SSI after THA & 9720 & $192 *$ & 1 year & $\begin{array}{l}\text { Infectious Diseases } \\
\text { Society of America } \\
\text { Criteria }\end{array}$ \\
\hline $\begin{array}{l}\text { Maradit } \\
\text { Kremers } \\
\text { et al. [23] }\end{array}$ & USA & 2002-2009 & $\begin{array}{l}\text { Retrospective } \\
\text { cohort }\end{array}$ & $\begin{array}{l}\text { Patients who underwent } \\
\text { primary or revision } \\
\text { TKA at Rochester } \\
\text { Mayo Clinic }\end{array}$ & $67 \cdot 7$ & $\begin{array}{l}\text { Claims-based risk } \\
\text { model } \\
\text { for TKA }\end{array}$ & SSI after TKA & 10451 & $192 *$ & 1 year & $\begin{array}{l}\text { Infectious Diseases } \\
\text { Society of America } \\
\text { Criteria }\end{array}$ \\
\hline $\begin{array}{l}\text { Tikhilov } \\
\text { et al. [24] }\end{array}$ & Russia & 2008-2012 & $\begin{array}{l}\text { Retrospective } \\
\text { cohort }\end{array}$ & $\begin{array}{l}\text { Patients treated for PJI } \\
\text { of the hip }\end{array}$ & $56 \cdot 1$ & NS & PJI recurrence & 217 & 78 & NR & NR \\
\hline
\end{tabular}

ATC, Anatomic, Therapeutic and Chemical Classification; CDC, Centre for Disease Control; HPRO, National Healthcare Safety Network surgical site infections risk model for hip arthroplasty; KPRO, National Healthcare Safety Network surgical site infections risk model for knee arthroplasty; NHSN, National Healthcare Safety Network; NR, not reported; NS, not stated; PJI, periprosthetic joint infection; SSI, surgical site infections; THA, total hip arthroplasty; TKA, total knee arthroplasty; ICD-10-AM, International Statistical Classification of Diseases and Related Health Problems, Tenth Revision, Australian Modification.

* Indicates the total number of SSIs for both THA and TKA. 
Table 2. Key characteristics of risk prediction scores for PJI included in the review

\begin{tabular}{|c|c|c|c|c|c|c|c|c|c|}
\hline $\begin{array}{l}\text { Lead author, } \\
\text { publication } \\
\text { date }\end{array}$ & $\begin{array}{l}\text { Name of } \\
\text { risk tool }\end{array}$ & $\begin{array}{l}\text { Statistical } \\
\text { model }\end{array}$ & Predictors used & $\begin{array}{l}\text { Number of } \\
\text { predictors }\end{array}$ & $\begin{array}{l}\text { Discrimination } \\
\text { (C-index) }\end{array}$ & $\begin{array}{l}\text { Calibration } \\
\text { (HL goodness- } \\
\text { of-fit test) }\end{array}$ & $\begin{array}{l}\text { Internal } \\
\text { validation }\end{array}$ & $\begin{array}{l}\text { External } \\
\text { validation }\end{array}$ & Performance comparison \\
\hline $\begin{array}{l}\text { Geubbels et al. } \\
{[17]}\end{array}$ & $\begin{array}{l}\text { THA-specific risk } \\
\text { model for SSI }\end{array}$ & $\begin{array}{l}\text { Logistic } \\
\text { regression }\end{array}$ & $\begin{array}{l}\text { Age, duration of } \\
\text { preoperative hospital stay, } \\
\text { postdischarge surveillance } \\
\text { and number of discharge } \\
\text { diagnoses }\end{array}$ & 4 & $0 \cdot 64$ & $\begin{array}{l}\text { Satisfactory } \\
\text { goodness of fit } \\
\text { reported }\end{array}$ & $\begin{array}{r}\text { Cross-validation } \\
\text { C-index }=0.62\end{array}$ & None & $\begin{array}{l}\text { Compared with the NNIS } \\
\text { index }(\mathrm{C} \text {-index }=0.56 \text {; } \\
P<0 \cdot 001)\end{array}$ \\
\hline Mu et al. [18] & HPRO & $\begin{array}{l}\text { Logistic } \\
\text { regression }\end{array}$ & $\begin{array}{l}\text { Age, anaesthesia, ASA, } \\
\text { procedure duration, type } \\
\text { of surgery (total primary, } \\
\text { partial primary, partial } \\
\text { revision, total revision), } \\
\text { bed size and trauma }\end{array}$ & 7 & $\begin{array}{l}0.66 \text { for SSI (superficial } \\
\text { incisional, deep } \\
\text { incisional and organ/ } \\
\text { space) } \\
0.67 \text { for deep incisional } \\
\text { and organ/space SSIs }\end{array}$ & NR & $\begin{array}{l}\text { Bootstrapping } \\
\text { resampling }\end{array}$ & $\begin{array}{l}\text { Externally } \\
\text { validated in } \\
\text { Lewallen et al. } \\
{[21]}\end{array}$ & $\begin{array}{l}\text { Compared with traditional } \\
\text { NHSN risk index (C-index } \\
=0 \cdot 61 \text { ) } \\
P \text {-value for comparison of } \\
\text { the two models }<0 \cdot 0001\end{array}$ \\
\hline Mu et al. [18] & KPRO & $\begin{array}{l}\text { Logistic } \\
\text { regression }\end{array}$ & $\begin{array}{l}\text { Age, anaesthesia, ASA, } \\
\text { procedure duration, } \\
\text { gender, type of surgery } \\
\text { (revision } v s . \text { primary), bed } \\
\text { size and trauma }\end{array}$ & 8 & $\begin{array}{l}0.64 \text { for SSI (superficial } \\
\text { incisional, deep } \\
\text { incisional and organ/ } \\
\text { space) } \\
0.65 \text { for deep incisional } \\
\text { and organ/space SSIs }\end{array}$ & NR & $\begin{array}{l}\text { Bootstrapping } \\
\text { resampling }\end{array}$ & $\begin{array}{l}\text { Externally } \\
\text { validated in } \\
\text { Lewallen et al. } \\
{[21]}\end{array}$ & $\begin{array}{l}\text { Compared with traditional } \\
\text { NHSN risk index (C-index } \\
=0 \cdot 60 \text { ) } \\
P \text {-value for comparison of } \\
\text { the two models }<0 \cdot 0001\end{array}$ \\
\hline $\begin{array}{l}\text { Paxton et al. } \\
{[16]}\end{array}$ & NS & $\begin{array}{l}\text { Cox } \\
\text { regression }\end{array}$ & $\begin{array}{l}\text { Age, sex, race, indication for } \\
\text { knee replacement, diabetes } \\
\text { and its complications, and } \\
\text { BMI }\end{array}$ & 6 & NR & $P=0.80$ & None & None & None \\
\hline $\begin{array}{l}\text { Berbari et al. } \\
\text { [19] }\end{array}$ & $\begin{array}{l}\text { Baseline Mayo PJI } \\
\text { risk score }\end{array}$ & $\begin{array}{l}\text { Logistic } \\
\text { regression }\end{array}$ & $\begin{array}{l}\text { BMI, prior other operation } \\
\text { on the index joint, prior } \\
\text { arthroplasty, } \\
\text { immunosuppression, ASA } \\
\text { score and procedure } \\
\text { duration }\end{array}$ & 6 & $\begin{array}{l}\text { Original: } 0 \cdot 722 \\
\text { Bias-corrected: } 0 \cdot 690\end{array}$ & $\begin{array}{l}\text { Satisfactory model } \\
\text { calibration }\end{array}$ & None & None & $\begin{array}{l}\text { Compared with traditional } \\
\text { NHSN risk index }(\mathrm{C} \text {-index } \\
=0.638 ; P<0.001)\end{array}$ \\
\hline $\begin{array}{l}\text { Berbari et al. } \\
\text { [19] }\end{array}$ & $\begin{array}{r}\text { 1-month-postsurgery } \\
\text { Mayo PJI risk score }\end{array}$ & $\begin{array}{l}\text { Logistic } \\
\text { regression }\end{array}$ & $\begin{array}{l}\text { BMI, prior other operation } \\
\text { on the index joint, prior } \\
\text { arthroplasty, } \\
\text { immunosuppression, ASA } \\
\text { score, procedure duration } \\
\text { and postoperative wound } \\
\text { drainage }\end{array}$ & 7 & $\begin{array}{l}\text { Original: } 0 \cdot 716 \\
\text { Bias-corrected: } 0 \cdot 680\end{array}$ & NR & None & None & $\begin{array}{l}\text { Compared with traditional } \\
\text { NHSN risk index }(\text { C-index } \\
=0.633 ; P<0.001)\end{array}$ \\
\hline Bozic et al. [20] & NS & $\begin{array}{l}\text { Logistic } \\
\text { regression }\end{array}$ & $\begin{array}{l}29 \text { comorbid conditions, } \\
\text { age, sex and } \\
\text { socioeconomic status }\end{array}$ & 32 & NR & NR & None & None & None \\
\hline $\begin{array}{l}\text { Lewallen et al. } \dagger \\
\text { [21] }\end{array}$ & HPRO & $\begin{array}{l}\text { Logistic } \\
\text { regression }\end{array}$ & $\begin{array}{l}\text { Age, anaesthesia, ASA, } \\
\text { procedure duration, type } \\
\text { of surgery (total primary, } \\
\text { partial primary, partial } \\
\text { revision, total revision), } \\
\text { bed size and trauma }\end{array}$ & 7 & $\begin{array}{l}0.695 \text { for SSI } \\
0.737 \text { for PJI }\end{array}$ & $\begin{array}{l}P=0.323 \text { for SSI } \\
P=0.606 \text { for PJI }\end{array}$ & N/A & N/A & $\begin{array}{l}\text { Modest improvement in } \\
\text { discrimination on addition } \\
\text { of morbid obesity and } \\
\text { diabetes mellitus to the } \\
\text { model } \\
\text { C-index }=0.706 \text { for SSI } \\
\text { C-index }=0.746 \text { for PJI }\end{array}$ \\
\hline
\end{tabular}


Table 2 (cont.)

\begin{tabular}{|c|c|c|c|c|c|c|c|c|c|}
\hline $\begin{array}{l}\text { Lead author, } \\
\text { publication } \\
\text { date }\end{array}$ & $\begin{array}{l}\text { Name of } \\
\text { risk tool }\end{array}$ & $\begin{array}{l}\text { Statistical } \\
\text { model }\end{array}$ & Predictors used & $\begin{array}{l}\text { Number of } \\
\text { predictors }\end{array}$ & $\begin{array}{l}\text { Discrimination } \\
\text { (C-index) }\end{array}$ & $\begin{array}{l}\text { Calibration } \\
\text { (HL goodness- } \\
\text { of-fit test) }\end{array}$ & $\begin{array}{l}\text { Internal } \\
\text { validation }\end{array}$ & $\begin{array}{l}\text { External } \\
\text { validation }\end{array}$ & Performance comparison \\
\hline $\begin{array}{l}\text { Lewallen et al. } \dagger \\
{[21]}\end{array}$ & KPRO & $\begin{array}{l}\text { Logistic } \\
\text { regression }\end{array}$ & $\begin{array}{l}\text { Age, anaesthesia, ASA, } \\
\text { procedure duration, } \\
\text { gender, type of surgery } \\
\text { (revision } v s . \text { primary), bed } \\
\text { size and trauma }\end{array}$ & 8 & $\begin{array}{l}0.592 \text { for SSI } \\
0.645 \text { for PJI }\end{array}$ & $\begin{array}{l}P=0.121 \text { for SSI } \\
P=0.072 \text { for } \mathrm{PJI}\end{array}$ & N/A & N/A & $\begin{array}{l}\text { Modest improvement in } \\
\text { discrimination on addition } \\
\text { of morbid obesity and } \\
\text { diabetes mellitus to the } \\
\text { model } \\
\text { C-index }=0.623 \text { for SSI } \\
\text { C-index }=0.669 \text { for PJI }\end{array}$ \\
\hline Inacio et al. [22] & RxRisk-V for THA & $\begin{array}{l}\text { Logistic } \\
\text { regression }\end{array}$ & $\begin{array}{l}42 \text { comorbid conditions plus } \\
\text { age, gender and primary } \\
\text { diagnoses for surgery }\end{array}$ & 45 & $0 \cdot 60$ & 0.793 & None & None & None \\
\hline Inacio et al. [22] & Elixhauser for THA & $\begin{array}{l}\text { Logistic } \\
\text { regression }\end{array}$ & $\begin{array}{l}30 \text { comorbid conditions plus } \\
\text { age, gender and primary } \\
\text { diagnoses for surgery }\end{array}$ & 33 & 0.59 & 0.744 & None & None & None \\
\hline Inacio et al. [22] & Charlson for THA & $\begin{array}{l}\text { Logistic } \\
\text { regression }\end{array}$ & $\begin{array}{l}17 \text { comorbid conditions plus } \\
\text { age, gender and primary } \\
\text { diagnoses for surgery }\end{array}$ & 20 & 0.58 & $0 \cdot 905$ & None & None & None \\
\hline Inacio et al. [22] & RxRisk-V for TKA & $\begin{array}{l}\text { Logistic } \\
\text { regression }\end{array}$ & $\begin{array}{l}42 \text { comorbid conditions plus } \\
\text { age, gender and primary } \\
\text { diagnoses for surgery }\end{array}$ & 45 & 0.57 & 0.057 & None & None & None \\
\hline Inacio et al. [22] & Elixhauser for TKA & $\begin{array}{l}\text { Logistic } \\
\text { regression }\end{array}$ & $\begin{array}{l}30 \text { comorbid conditions plus } \\
\text { age, gender and primary } \\
\text { diagnoses for surgery }\end{array}$ & 33 & 0.58 & $0 \cdot 827$ & None & None & None \\
\hline Inacio et al. [22] & Charlson for TKA & $\begin{array}{l}\text { Logistic } \\
\text { regression }\end{array}$ & $\begin{array}{l}17 \text { comorbid conditions plus } \\
\text { age, gender and primary } \\
\text { diagnoses for surgery }\end{array}$ & 20 & 0.56 & 0.513 & None & None & None \\
\hline $\begin{array}{l}\text { Maradit } \\
\text { Kremers et al. } \\
\text { [23] }\end{array}$ & $\begin{array}{c}\text { Claims-based risk } \\
\text { model for THA }\end{array}$ & $\begin{array}{l}\text { Cox } \\
\text { regression }\end{array}$ & $\begin{array}{l}\text { Age, sex, type of surgery } \\
\text { (primary } v . \text { revision), and } \\
16 \text { individual Charlson } \\
\text { index comorbidities }\end{array}$ & 19 & $\begin{array}{l}\text { Original: } 0.662 \\
\text { Bias-corrected: } 0.629\end{array}$ & $\begin{array}{l}\text { Reported as } \\
\text { 'reasonably } \\
\text { calibrated' }\end{array}$ & $\begin{array}{l}\text { Bootstrap } \\
\text { resampling }\end{array}$ & None & $\begin{array}{l}\text { On addition of four clinical } \\
\text { predictors (morbid obesity, } \\
\text { prior surgeries on the same } \\
\text { joint, ASA score and length } \\
\text { of operative time) } \\
\text { Original C-index: } 0 \cdot 706 \\
\text { Bias-corrected C-index: } 0 \cdot 665 \\
\text { Difference in C statistic: } 0 \cdot 043 \\
(0 \cdot 012-0.074) \\
\text { Aggregated IDI: } 0 \cdot 37 \%(0 \cdot 12- \\
0 \cdot 62 \%)\end{array}$ \\
\hline $\begin{array}{l}\text { Maradit } \\
\text { Kremers et al. } \\
\text { [23] }\end{array}$ & $\begin{array}{c}\text { Claims-based risk } \\
\text { model for TKA }\end{array}$ & $\begin{array}{l}\text { Cox } \\
\text { regression }\end{array}$ & $\begin{array}{l}\text { Age, sex, type of surgery } \\
\text { (primary } v s . \text { revision), and } \\
16 \text { individual Charlson } \\
\text { index comorbidities }\end{array}$ & 19 & $\begin{array}{l}\text { Original: } 0 \cdot 621 \\
\text { Bias-corrected: } 0.585\end{array}$ & $\begin{array}{l}\text { Reported as } \\
\text { 'reasonably } \\
\text { calibrated' }\end{array}$ & $\begin{array}{l}\text { Bootstrap } \\
\text { resampling }\end{array}$ & None & $\begin{array}{l}\text { On addition of four clinical } \\
\text { predictors (morbid obesity, } \\
\text { prior surgeries on the same } \\
\text { joint, ASA score, and length } \\
\text { of operative time) } \\
\text { Original C-index: } 0.648 \\
\text { Bias-corrected C-index: } 0.606 \\
\text { Difference in C statistic: } \\
0.027(0.007-0.047) \\
\text { Aggregated IDI: } 0.09 \% \\
(-0.02 \% \text { to } 0.21 \%)\end{array}$ \\
\hline
\end{tabular}




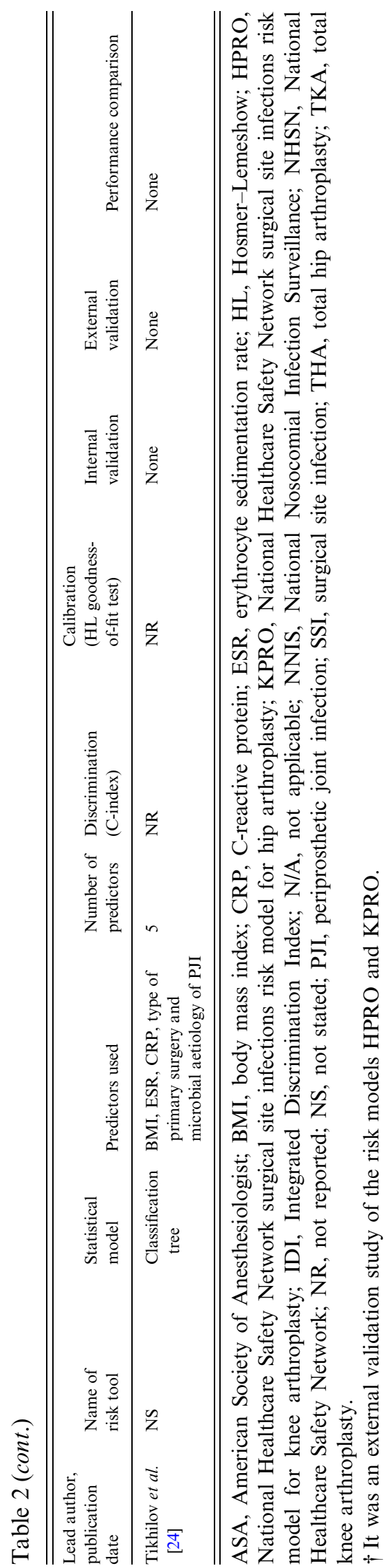

reports of any validation and performance comparisons made. A total of 16 risk scores were described in the nine eligible studies. Five of these scores had separate models for hip and knee replacement patients [18, 22, 23]. Four studies described the development of two or more risk scores [18, 19, 22, 23]. All 16 risk scores were derivations of risk models on a base population and two of them were also externally validated on new populations [21]. Except for one study that developed the risk score based on a cohort recruited prospectively for the surveillance of SSIs [17], all studies used datasets retrospectively that had been established for different purposes. Except for the scores that were developed in both knee and hip replacement patients, the component predictors varied from score to score. However, age, sex and type of primary surgery featured in the majority of risk scores. Except for one score that was mainly based on invasive data such as ESR (erythrocyte sedimentation rate), CRP (C-reactive protein) and microbial aetiology [24], all scores were based on data that can be assessed non-invasively such as demographics, anthropometrics, medical and surgical histories, and surgical procedures. The number of component variables in a single score ranged from 4 to 45 $(n=16$, median 19, interquartile range 6.5-32.5). Seven out of the 16 risk scores had 10 or fewer components. Of the 16 risk scores, 15 used regression techniques (logistic or Cox) to develop the score and one used a classification tree [24].

\section{Model diagnostics}

Except for three studies (comprising of three risk scores) $[16,20,24]$, the C-statistic was reported for 13 risk scores. The C-index ranged from 0.56 to 0.74 . Only three risk scores were reported to have a discriminative ability of $>0.70$ and these were the baseline Mayo and 1-month-postsurgery Mayo PJI risk scores as reported by Berbari et al. [19] and HPRO which was externally validated by Lewallen et al. [21], Calibration measures were presented for 11 risk scores (including the baseline Mayo PJI risk score) and each was reported to have satisfactory model calibration. Two studies did not report on any measures of discrimination or calibration [20, 24] (Table 2).

\section{Model validation}

Only five of the risk scores were validated internally using resampling techniques such as bootstrapping and cross-validation $[17,18,23]$. These included (i) a 
total hip arthroplasty (THA)-specific risk model for SSI, developed using data collected from 62 acute care hospitals within the Dutch surveillance network for nosocomial infections [17]; (ii) the HPRO and KPRO [18] and (iii) claims-based risk models for THA and total knee arthroplasty (TKA) [23]. Only the HPRO and KPRO risk scores were externally validated using an independent dataset in a different study [18]. Although the HPRO score performed better in the external cohort compared with the internal validation cohort, the KPRO risk score performed much less well when tested on the external cohort compared with the internal cohort (Table 2).

\section{Performance comparisons}

The performances of five risk scores were compared with existing models in three studies [17-19]. Geubbels et al. compared the predictive performance of their newly developed THA-specific risk score for SSI with the NNIS (National Nosocomial Infection Surveillance) system risk index (which incorporates three risk factors of equal weight namely wound contamination class, American Society of Anesthesiologists (ASA) score, and duration of surgery), and reported better predictive performance for the new risk score (C-index: 0.64 vs. 0.56; $P<0.001$ ) [17]. Mu et al. also reported statistically significantly better performances for the HPRO and KPRO risk scores when compared with the traditional NHSN SSI risk model, though the C-statistics were generally low $(<0 \cdot 70)$ [18]. The baseline Mayo and 1-month-postsurgery Mayo PJI risk scores also performed well compared with the traditional NHSN SSI risk score (C-index: 0.72 vs. $0.64 ; P$ $<0.001)$ and (C-index: 0.72 vs. $0.63 ; P<0.001)$, respectively [19]. Two studies assessed the incremental prognostic value of adding additional risk factors to their existing models [21, 23]. Lewallen et al. externally validated the HPRO and KPRO risk scores and reported that addition of information on morbid obesity and diabetes mellitus to each score modestly improved discrimination [21]. On addition of four clinical risk factors (morbid obesity, prior non-arthroplasties on the same joint, ASA score and operative time) to their claimsbased risk models for THA and TKA, Maradit Kremers et al. reported improved performance (by C-statistics) for both models, though the THA model showed better performance than the TKA model [23]. There was however no noticeable improvement in calibration for both models. Finally, whiles there was an improvement in IDI (Integrated Discrimination Index) for the THA score, no significant improvement was seen for the TKA score: $0 \cdot 37 \%(0 \cdot 12 \%$ to $0 \cdot 62 \%)$ and $0.09 \%(-0.02 \%$ to $0.21 \%)$, respectively.

\section{Clinical evaluation of risk scores}

None of the studies described the evaluation of the clinical effectiveness of a score in an intervention study or as part of an impact study aimed at changing patient outcomes.

\section{DISCUSSION}

\section{Key findings}

Using systematic review methods, we have reported the first overview of available risk assessment scores for SSI or PJI following joint replacement. Based on established quality criteria for risk scores [25, 26], none of the risk scores in our review were judged to be promising for use in clinical settings or public health practice, except for the HPRO. The HPRO is a procedure-specific risk score which was adapted from the traditional NHSN risk index using NHSN data and its purpose is for predicting SSI or PJI within 1 year of hip replacement [18]. The HPRO was found to perform better than the traditional NHSN risk index and external validation in an independent cohort showed high discriminative ability [21]. The HPRO also showed higher accuracy for predicting PJI compared with SSI. The data also show that risk prediction models for SSI or PJI have only been developed over the past 5 years. Of the 16 risk scores identified, only seven had 10 or few components included in the final score, with a number of scores having between 30 and 45 components. Although all 11 risk scores reporting calibration measures exhibited satisfactory calibration, only three of these risk scores were reported to have a discriminative ability of $>0 \cdot 70$. Of all 16 risk scores, HPRO and KPRO were the only risk scores externally validated in an independent population. Quality assessment of the risk scores' development and validation criteria showed all scores to have a high risk of bias. This was mainly due to the methodology used in assessment of predictors and outcomes, inappropriate handling of missing data, and lack of external validation.

\section{Explanations and implications of findings}

Our findings highlight the limited evidence available on appropriate risk scores for predicting SSI or PJI after joint replacement. Given the absence of an 
ideal risk score which can be used in a routine clinical setting, it appears that the potential value of risk scores in preventing SSI or PJI may have been underestimated in orthopaedic practice. The findings also highlight the use of poor methodology in the development of some of these risk scores. Although crosssectional study designs were not included, the included studies were not free from bias and confounding. The majority of the designs were based on retrospective cohorts instead of prospective cohort designs, which are ideal for risk score modelling as predictor information can be ascertained blindly in relation to the outcome or disease [13]. None of the risk scores was developed in a cohort recruited for this sole purpose, which introduced an inherent selection bias. A key methodological issue was the absence of clear and detailed reporting of the treatment of missing data in all studies, which is of utmost importance prior to the development of risk scores [12]. Included studies used complete case analysis in the presence of missing data, which does not represent the entire population and reduces the sample size [13]. It has been shown that risk scores that use multiple imputation, produce more valid results and have better discrimination than tools that ignore such additional analyses [27]. There were also concerns with usability of the risk scores, as the majority of the risk scores had more than 10 variables. It is recognised that the simplicity of the model is an important criteria for developing clinically useful risk scores [28, 29]. Evidence suggests that complex models are more likely to provide overoptimistic predictions, especially when extensive variable selection has been performed [30]. Only five of the risk scores were validated internally using resampling methods, which are techniques which give a good indication of how optimistic the risk score may be [31]. Although internal validation is helpful, it cannot provide information on the model's performance elsewhere or its generalisability. Before a risk prediction tool can be used in clinical practice or in real-world settings, evaluation of its generalisability (or transportability) requires data from elsewhere - also known as external validation [12]. However, only two risk scores were externally validated in our sample [21]. Finally, none of the risk scores was reported to have been used in an impact study aimed at changing patient outcomes. Before a risk score can be implemented, a vital criterion that needs to be fulfilled is its impact on clinical practice [12]. Among the identified risk scores, only the HPRO was found to be potentially promising for use in a clinical setting.
However, it cannot be considered ready for use as its clinical effectiveness is still yet to be evaluated. The unavailability of appropriate existing risk scores for use in the clinical setting is extremely concerning. To add to this challenge is the lack of established uniform criteria for the diagnosis of infection especially PJI, which actually makes it difficult to conduct diagnostic or risk prediction studies for infection. Although hip and knee replacements are successful elective procedures, with SSIs or PJIs being rare complications of these procedures [3, 32]; the incidence of these infections will increase in conjunction with growing healthcare burden due to osteoarthritis [33] and a predicted large rise in the numbers of arthroplasty procedures $[34,35]$. To meet this challenge, there should be a clinical drive towards identification of individuals at high risk of SSIs or PJIs using risk prediction engines. The current findings should stimulate research groups to develop and evaluate appropriate infection outcome-specific risk prediction algorithms using robust methodology. The clinical effectiveness of the HPRO also needs to be evaluated before it is implemented. Within our 5 year INFORM (INFection ORthopaedic Management) Programme, the aim is to develop and establish optimum strategies for the prevention and treatment of PJIs within the UK National Health System [36], and which may include the development of appropriate risk prediction engines when the data allows.

\section{Study strengths and limitations}

To the best of our knowledge, this is the first systematic review to identify limited progress in the development and validation of risk prediction models for SSI or PJI following joint replacement, using robust systematic methodology. It is also the first review to assess the validity of existing risk scores based on risk of bias and applicability. Our search strategy was comprehensive and spanned multiple databases, making it unlikely that any relevant study was missed. There was variation in the definition of SSIs in the included studies, which did not allow for a head-to-head comparison of risk scores across studies. We were unable to harmonise data from contributing studies to perform a quantitative analysis, due to the heterogeneity in study designs and populations, predictors used, model types, and measures reported. Even though we tried to present the data as robustly as possible using established criteria, our conclusions might be limited due to the 
quality of published research and the large variability across study characteristics and methodologies.

\section{CONCLUSION}

In conclusion, available risk scores to predict SSI or PJI have been developed using poor methodology and have several limitations. The majority of these risk scores have not been externally validated and are not ideal for use in clinical settings. The HPRO is the only risk prediction tool identified to show some promise for use in a clinical setting (based on its predictive performance and having some external validation); however, it needs further validation using new data and its clinical effectiveness should be evaluated using a RCT design. A potentially effective way of tackling the increasing incidence of SSIs is early and accurate identification of individuals at high risk using established risk prediction scores, an approach which has been very effective in the area of CVD prevention. Further research is urgently warranted within the field to develop and test appropriate outcome-specific risk prediction tools.

\section{SUPPLEMENTARY MATERIAL}

The supplementary material for this article can be found at https://doi.org/10.1017/S0950268817000486

\section{ACKNOWLEDGEMENTS}

This publication is part of the INFection OR thopaedic Management (INFORM) Programme. As such it benefits from involvement of the whole INFORM team. The INFORM team includes: Simon Strange, Setor Kunutsor, Kirsty Garfield, Erik Lenguerrand, Rachael Gooberman-Hill, Drew Moore, Amanda Burston, Joanne Simon, Garry King, Michael Whitehouse, Vikki Wylde, Andrew Beswick, Ashley Blom, Sian Noble, Athene Lane, Fran Carroll (Musculoskeletal Research Unit, School of Clinical Sciences, University of Bristol, Southmead Hospital, Southmead Road, Bristol, BS10 5NB, UK); Sian Noble, Athene Lane, Fran Carroll (School of Social and Community Medicine, University of Bristol, Bristol, BS8 2PS, UK); Jason Webb, Alasdair MacGowan (North Bristol NHS Trust, Southmead Hospital, Bristol, BS10 5NB, UK); Stephen Jones (Cardiff and Vale University Health Board, Longcross Street, Cardiff, CF24 0SZ, UK); Adrian Taylor (Oxford University Hospitals NHS Trust, John Radcliffe Hospital,
Oxford OX3 9DU, UK); Paul Dieppe (University of Exeter, Medical School, Exeter, EX1 2LU, UK); Andrew Toms, Matthew Wilson (Royal Devon and Exeter NHS Foundation Trust, Newcourt House, Exeter, EX2 7JU, UK); Ian Stockley (Sheffield Teaching Hospitals NHS Trust, Northern General Hospital, Sheffield, S5 7AU, UK); Ben Burston, John-Paul Whittaker (The Robert Jones and Agnes Hunt Orthopaedic Hospital NHS Foundation Trust, Oswestry, Shropshire, SY10 7AG, UK); Tim Board (Wrightington, Wigan and Leigh NHS Foundation Trust, Apple Bridge, Wigan, Lancashire, WN6 9EP, UK); and all the surgeons and nurses from the collaborating centres. This article presents independent research funded by the National Institute for Health Research (NIHR) under its Programme Grants for Applied Research program (RP-PG-1210-12005). The views expressed in this article are those of the authors and not necessarily those of the NHS, the NIHR, or the Department of Health.

\section{DECLARATION OF INTEREST}

M.R.W. reports grants from the National Institute of Health Research during the conduct of the study; grants from British Orthopaedic Association, which were outside the submitted work.

\section{REFERENCES}

1. Mangram AJ, et al. Guideline for prevention of surgical site infection, 1999. Centers for Disease Control and Prevention (CDC) Hospital Infection Control Practices Advisory Committee. American Journal of Infection Control 1999; 27(2): 97-132; quiz 133-134; discussion 196.

2. Peersman G, et al. Infection in total knee replacement: a retrospective review of 6489 total knee replacements. Clinical Orthopaedics and Related Research 2001; 392: 15-23.

3. Kurtz SM, et al. Prosthetic joint infection risk after TKA in the Medicare population. Clinical Orthopaedics and Related Research 2010; 468(1): 52-56.

4. Hunter G, Dandy D. The natural history of the patient with an infected total hip replacement. The Journal of Bone and Joint Surgery. British Volume 1977; 59-B(3): 293-297.

5. Cahill JL, et al. Quality of life after infection in total joint replacement. Journal of Orthopaedic Surgery (Hong Kong) 2008; 16(1): 58-65.

6. Andersson AE, et al. Patients' experiences of acquiring a deep surgical site infection: an interview study. American Journal of Infection Control 2010; 38(9): 711-717. 
7. Kallala RF, et al. Financial analysis of revision knee surgery based on NHS tariffs and hospital costs: does it pay to provide a revision service? The Bone \& Joint Journal 2015; 97-B(2): 197-201.

8. Vanhegan IS, et al. A financial analysis of revision hip arthroplasty: the economic burden in relation to the national tariff. The Journal of Bone and Joint Surgery. British Volume 2012; 94(5): 619-623.

9. Kurtz SM, et al. Future young patient demand for primary and revision joint replacement: national projections from 2010 to 2030. Clinical Orthopaedics and Related Research 2009; 467(10): 2606-2612.

10. Kunutsor SK, et al. Patient-related risk factors for periprosthetic joint infection after total joint arthroplasty: a systematic review and meta-analysis. PLOS ONE 2016; 11(3): e0150866.

11. Triantafyllopoulos G, et al. Patient, surgery, and hospital related risk factors for surgical site infections following total hip arthroplasty. Scientific World Journal 2015; 2015: 979560.

12. Steyerberg EW, et al. Prognosis Research Strategy (PROGRESS) 3: prognostic model research. PLoS Medicine 2013; 10(2): e1001381.

13. Ensor J, et al. Systematic review of prognostic models for recurrent venous thromboembolism (VTE) posttreatment of first unprovoked VTE. BMJ Open 2016; 6(5): e011190.

14. Cook NR, et al. Comparison of the Framingham and Reynolds risk scores for global cardiovascular risk prediction in the multiethnic Women's Health Initiative. Circulation 2012; 125(14): 1748-1756, S1-11.

15. Moher D, et al. Preferred reporting items for systematic reviews and meta-analyses: the PRISMA statement. PLoS Medicine 2009; 6(7): e1000097.

16. Paxton EW, et al. Predicting the risk of deep infections following total knee replacement: a pragmatic, prognostic risk score. American Journal of Epidemiology 2011; 173: S19-S19.

17. Geubbels EL, et al. Improved risk adjustment for comparison of surgical site infection rates. Infection Control and Hospital Epidemiology 2006; 27(12): 1330-1339.

18. Mu Y, et al. Improving risk-adjusted measures of surgical site infection for the national healthcare safety network. Infection Control and Hospital Epidemiology 2011; 32(10): 970-986.

19. Berbari EF, et al. The Mayo prosthetic joint infection risk score: implication for surgical site infection reporting and risk stratification. Infection Control and Hospital Epidemiology 2012; 33(8): 774-781.

20. Bozic KJ, et al. Estimating risk in Medicare patients with THA: an electronic risk calculator for periprosthetic joint infection and mortality. Clinical Orthopaedics and Related Research 2013; 471(2): 574-583.

21. Lewallen $\mathbf{L W}$, et al. External validation of the national healthcare safety network risk models for surgical site infections in total hip and knee replacements. Infection
Control and Hospital Epidemiology 2014; 35(11): 1323-1329.

22. Inacio MC, et al. Predicting infections after total joint arthroplasty using a prescription based comorbidity measure. The Journal of Arthroplasty 2015; 30(10): 1692-1698.

23. Maradit Kremers H, et al. Do claims-based comorbidities adequately capture case mix for surgical site infections? Clinical Orthopaedics and Related Research 2015; 473(5): 1777-1786.

24. Tikhilov R, et al. Risk factors and a prognostic model of hip periprosthetic infection recurrence after surgical treatment using articulating and non-articulating spacers. International Orthopaedics 2016; 40(7): 1381-1387.

25. Altman DG, et al. Prognosis and prognostic research: validating a prognostic model. British Medical Journal 2009; 338: b605.

26. Noble D, et al. Risk models and scores for type 2 diabetes: systematic review. British Medical Journal 2011; 343: $\mathrm{d} 7163$.

27. Janssen KJ, et al. Development and validation of clinical prediction models: marginal differences between logistic regression, penalized maximum likelihood estimation, and genetic programming. Journal of Clinical Epidemiology 2012; 65(4): 404-412.

28. Wyatt JC, Altman DG. Commentary: prognostic models: clinically useful or quickly forgotten? British Medical Journal 1995; 311(7019): 1539-1541.

29. Moons KG, et al. Prognosis and prognostic research: what, why, and how? British Medical Journal 2009; 338: b375.

30. Sauerbrei W. The use of resampling methods to simplify regression models in medical statistics. Journal of the Royal Statistical Society: Series C (Applied Statistics) 1999; 48(3): 313-329.

31. Steyerberg EW, et al. Internal and external validation of predictive models: a simulation study of bias and precision in small samples. Journal of Clinical Epidemiology 2003; 56(5): 441-447.

32. Dale $\mathbf{H}$, et al. Increasing risk of revision due to deep infection after hip arthroplasty. Acta Orthopaedica 2009; 80(6): 639-645.

33. Cross M, et al. The global burden of hip and knee osteoarthritis: estimates from the global burden of disease 2010 study. Annals of the Rheumatic Diseases 2014; 73 (7): 1323-1330.

34. Kurtz S, et al. Projections of primary and revision hip and knee arthroplasty in the United States from 2005 to 2030. The Journal of Bone and Joint Surgery American Volume 2007; 89-A(4): 780-785.

35. Patel A, et al. The epidemiology of revision total knee and hip arthroplasty in England and Wales: a comparative analysis with projections for the United States. A study using the National Joint Registry dataset. The Bone \& Joint Journal 2015; 97-B(8): 1076-1081.

36. Bellamy K, Rousseau SA, Gardner PS. The development of an $\mathrm{M}$ antibody capture ELISA for rubella IgM. Journal of Virological Methods 1986; 14(3-4): 243-251. 